\title{
LS 区存在对光流敏感细胞
}

\author{
谢晓晖 王磊 吕海东王连红 才云程
}

(中国科学院生物物理研究所视觉信息加工开放实验室, 北京 100101)

\section{关键词 光流 LS 区 视觉信息加工 本体运动}

猫视皮层 LS( Lateral supersylvian) 区细胞的感受野面积远大于初级视皮层细胞, 对运动光 棒有良好的反应, 约有 $80 \%$ 的细胞对方向敏感 ${ }^{[1]}$, 主要处理运动信息. 有人认为它相当于猴 子的 MT (Middle temporal) 区. MT 区的作用是将来自初级视区的信息输入进行加工, 进而输 出给 MST (Middle superior temporal) 等高级视区, 最终形成对旋转、收缩/扩张、平移、形变等由 本体运动引入的大范围较复杂的光流信息的反应 ${ }^{[2,3]}$. 尚不清楚猫视皮层是否存在能对较复 杂光流信息反应的高级脑区, 对光流信息加工方式是否与猴相同. 基于猫 LS 区细胞感受野 网膜位置与最佳运动方向关系的研究, Sherk 等认为, LS 区细胞可能参与对光流刺激的信息 加工 ${ }^{[4]}$. 然而, 直接用光流刺激进行的研究, 在猫中尚未见报道. 我们采用旋转、收缩/扩张、 平动等刺激模式研究, 结果显示 LS 区一些细胞对旋转、收缩/扩张等光流刺激敏感.

\section{1 材料与方法}

实验数据收集于 4 只成年健康家猫, 动物准备、记录和数据采集与先前的方法相同 ${ }^{[1]}$. 视 觉刺激是由 $0.5^{\circ}$ 大小, 间距约为 $2.5^{\circ}$ 的二维光点组成, 光点呈同心圆分布, 每个圆周上有 8 个 光点, 在直径 $5 \sim 15^{\circ}$ 的圆环内约有 5 个圆周. 光点运动速度为 $10 \sim 50^{\circ} \%$. 在大多数情况下, 刺激由暗背景 (光量度为 $0.5 \mathrm{~cd} / \mathrm{m}^{2}$ ) 和亮光点群 (光量度为 $48 \mathrm{~cd} / \mathrm{m}^{2}$ ) 组成. 实验中具体参数 可调以期达到最佳刺激. 光点运动的不同安排组成不同的刺激模式. 平动刺激由同向同速运 动的光点构成; 旋转刺激由沿切线方向运动的光点构成, 又分为顺时针运动和逆时针运动; 收 缩/扩张刺激由沿法线方向运动的光点组成, 由运动方向的不同构成扩张或收缩刺激.

\section{2 结果}

实验中研究了 4 只猫 127 个细胞的反应, 几乎所有细胞都对平动刺激反应或受其抑制, 其 中有 48 个细胞对旋转刺激敏感, 53 个细胞对收缩/扩张刺激敏感. 图 1 显示了其中 4 个细胞 对不同刺激的反应情况. 48 个对旋转刺激反应较好细胞中, $36 \%$ 对顺时针旋转反应比对逆时 针旋转反应强, $26 \%$ 对逆时针旋转反应比对顺时针反应强, $38 \%$ 对顺、逆时针反应都较好. 53 个对收缩/扩张刺激反应好细胞中, $62 \%$ 对扩张的反应强于对收缩的反应, $14 \%$ 对收缩反应强 于对扩张的反应, $24 \%$ 对扩张和收缩反应皆较好.

比较细胞在 3 种不同视觉刺激下的刺激后时间直方图 (Poststimulus time histogram, PSTH) : (1) 光点群的平动, (2) 光点群的旋转 (先顺时针旋转, 后逆时针旋转), (3) 光点群的收 缩/扩张 (先扩张后收缩). 图 2 左图显示了两个细胞的反应, (a) 图中细胞受平动刺激的抑制, 表现为自发活动的减少; 用收缩/扩张刺激, 细胞对扩张刺激反应较强. (b)图中细胞尽管受平 动刺激抑制, 但对旋转刺激, 则顺时针旋转刺激引发较强的兴奋反应. 图 2 右图用以上运动刺 

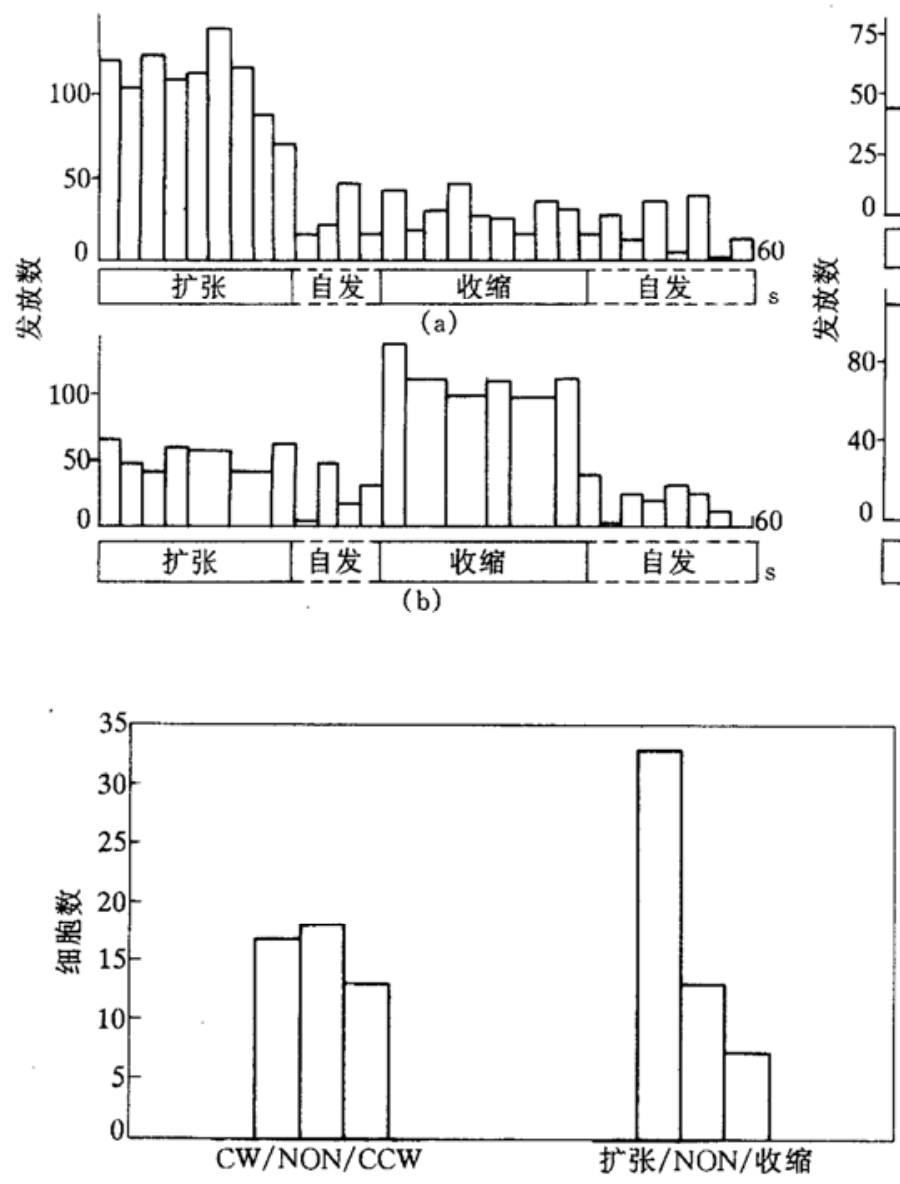

(e)

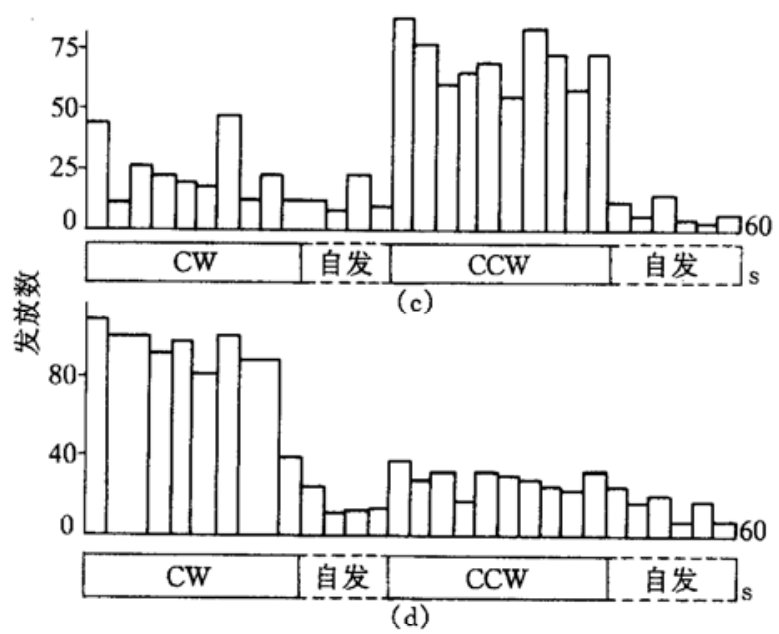

图 1 LS 区一些细胞对光流刺激敏 感, 图中显示了 4 个典型细胞的反应。 (a) 中细胞对扩张刺激敏感; (b) 中细胞对收 缩刺激敏感; (c) 中细胞对逆时针旋转敏感; (d) 中细胞对顺时针旋转敏感; (e) 图显示了 以上 4 种细胞数的统计分布. 图中 $\mathrm{CW}$ 表 示顺时针旋转刺激, $\mathrm{CCW}$ 表示逆时针旋转 刺激. (e) 图中 NON 表示对顺、逆时针旋 转或收缩、扩张刺激反应无偏好细胞

激的一半作为对照, 调节其亮度使屏的平均亮度不变. 这种刺激虽然也作旋转、收缩/扩张, 但 仅是局部光点的运动刺激, 失去了原刺激结构的整体特征. 由图可见, 这两种视觉刺激引发的 反应都远小于整体光流刺激诱发的反应, 且反映不出顺、逆时旋转和收缩/扩张上的差异. 这 些结果提示这些细胞可能对刺激的整体特征反应, 而不是感受野内视觉刺激反应的简单加和.

\section{3 讨论}

在本实验中, 我们首次发现猫 LS 区有些细胞能对旋转、收缩/扩张等光流刺激有较好反 应, 且这些细胞可能对光流刺激的整体特征敏感, 而不仅仅是感受野内不同光点反应的简单加 和, 说明 LS 区细胞确实参与加工视觉刺激的整体运动信息. LS 区的一些细胞对较大范围视 觉刺激敏感, 对刺激的具体形状、组成光点的大小并不敏感, 这提示与猴 MST 类似 ${ }^{[2]}$, 猫 LS 区细胞对光流信息的加工也与本体运动相关. 图 1 中细胞对光流成分刺激的长时间持续反应 也说明了这一点. 细胞对旋转方式下顺、逆时针的不同偏好, 以及对收缩/扩张方式下收缩、扩 张的不同反应与猴 MST 上的结果是一致的. 另外, 大部分 LS 区细胞对平动反应较好, 这则 与 MT 细胞的性质类似 ${ }^{[5]}$.

一些 LS 区细胞对光流刺激敏感有着重要的功能意义. 细胞对收缩/扩张的不同偏好可能参 与判别 time-to-collision 以及自身运动的方向 ${ }^{[5]}$; 细胞对旋转的不同偏好与动物头、眼的转动相关. LS 区一些细胞能对视觉刺激的整体特征敏感, 参与识别动物与外部环境间的相对运动而其对刺 

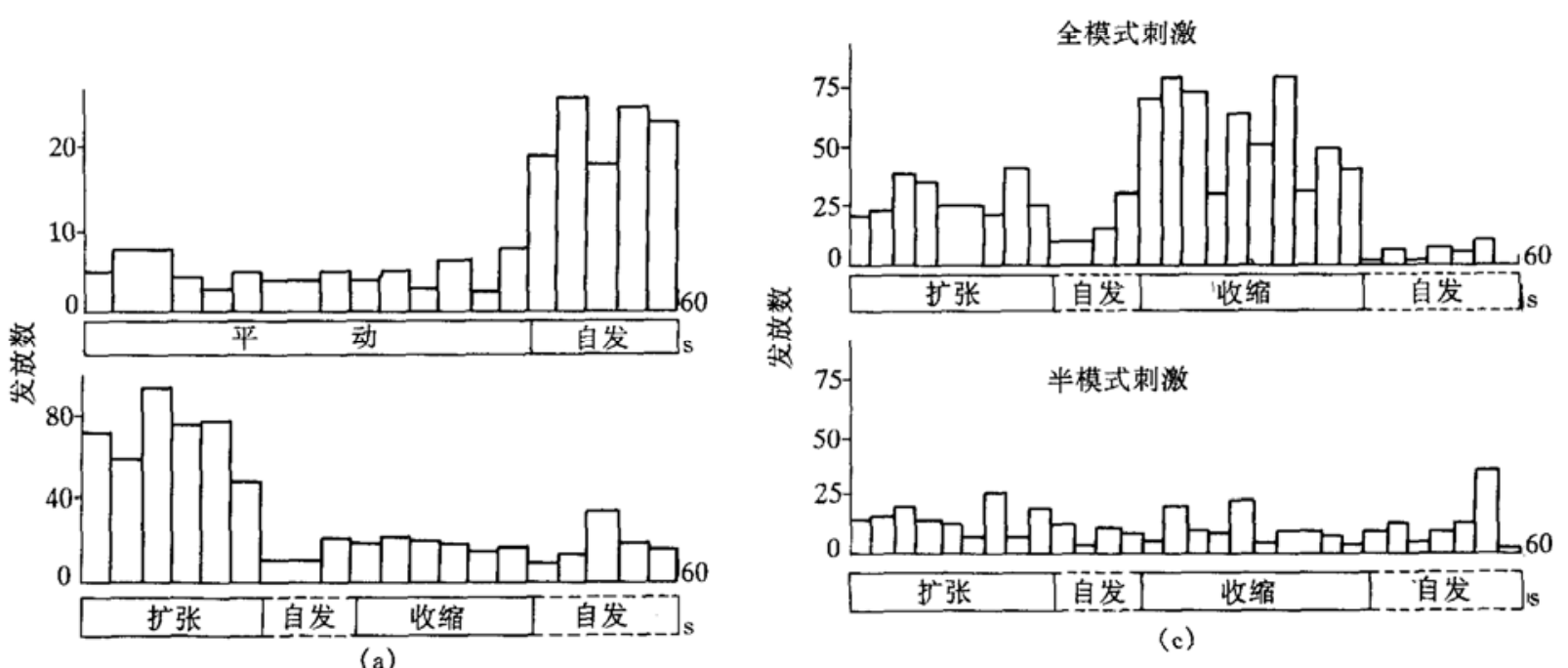

(a)

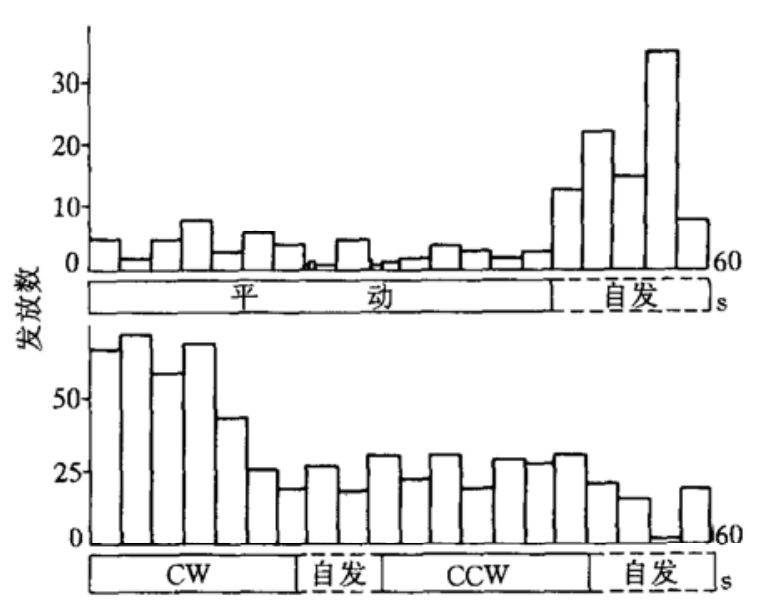

(b)

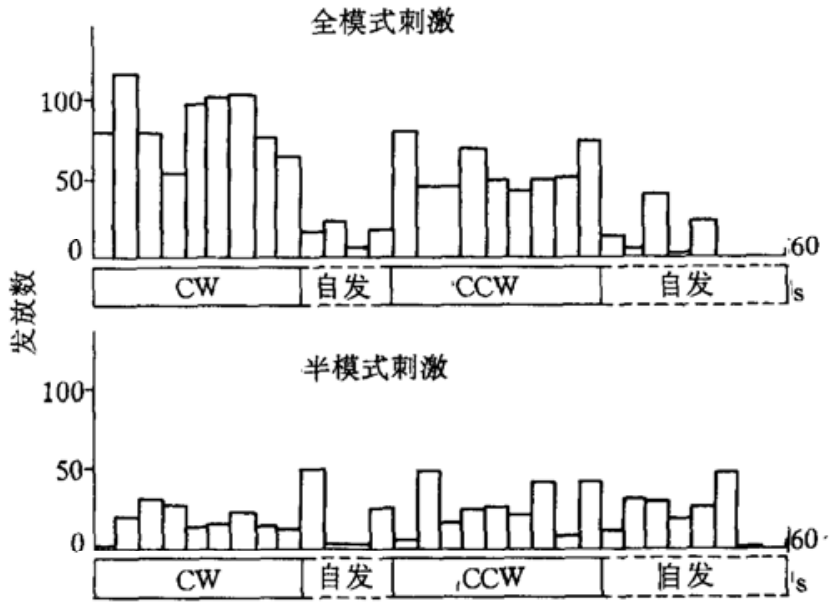

(d)

图 2 LS 区 4 个细胞在不同刺激下的反应

(a)、(b) 中细胞皆受平动抑制, 但却分别对扩张、顺时针旋转敏感; (c)、(d) 中细胞对光流的半部刺激反应较弱

激的形状、刺激的构成等具体参数并不敏感,这种信息加工方式与功能上的需要是吻合的.

LS 区对旋转刺激敏感细胞中, 顺时针、逆时针旋转不同偏好细胞数分布均匀, 而对收缩/ 扩张刺激敏感细胞中, 对扩张刺激反应较好细胞占多数. 这可能是因为动物本体运动并无顺 时、逆时旋转优势上的差异, 因而两种细胞数分布均匀. 但是, 由于动物多作前进运动, 因而对 扩张刺激反应较好的细胞比对收缩反应敏感的细胞多.

本实验中发现 LS 区细胞对收缩/扩张刺激的反应较强, 但猴 MST 细胞收缩/扩张反应相 对较弱 ${ }^{[2,5]}$. 这可能是因为猫主要在地面行动, 而猴主要树栖, 由于功能上的不同需要而导致 细胞反应上的差异. 另外, 我们注意到大部分 LS 细胞对旋转刺激和收缩/扩张刺激皆有较好 反应, 而 MST 细胞一般只对其中一种刺激选择反应 ${ }^{[2]}$.

\section{参考文献}

1 Wang Y, Wang L, Li B, et al. How is direction selectivity organized in the extrastriate visual area PMLS of the cat? Neuro Report, 1995, 6:1969 1974 
【研究简报】

\title{
小麦 POD 同工酶谱蛋白条带中硒的 中子活化分析
}

\author{
孙景信(1) 王玉琦 (1) 徐云 (2) 王子键 (2) 彭 安 ${ }^{(2)}$
}

(1)中国科学院高能物理研究所, 北京 100080; (2)中因科学院生态环境研究中心, 北京 100085)

\section{关键词 小麦 过氧化物酶同工酶 硒 中子活化分析}

硒是一种生命必需的微量元素 ${ }^{[1]}$. Rotruck 等发现硒是谷胱甘胝过氧化物酶 (GSH-Px)的 活性组分, 从而确认了 GSH-Px 是哺乳动物中发现的第一个硒酶 ${ }^{[2]}$. 此后, 对硒的代谢和功能 作用, 以及是否还存在其他的含硒酶或蛋白等的研究一直十分活跃 ${ }^{[3]}$. 到目前为止, 已经在 哺乳动物和微生物中陆续发现了一系列的含硒酶, 如动物体中的磷脂氢过氧物谷胱甘肽过氧 化物酶 (PHG-Px)、I 型硒甲状腺 $5^{\prime}$-脱碘酶等, 以及微生物中的甲酸脱氢酶、甘氨酸还原酶等 等. 然而, 对植物中的含硒蛋白或酶的研究甚少.

过氧化物酶 (Peroxidase, POD) 是植物体内最重要的氧化还原酶类之一. POD 具有多种 同工酶, 对外界环境反应十分敏感。在逆境条件下, POD 的活性往往增强, 同工酶谱发生变 化. 在植物的生长、发育和成熟过程中起十分重要的生理功能作用.

由于植物体内硒含量很低, 在经过生化分离后得到的 POD 同工酶蛋白条带中硒的含量则 更少, 一般在 ng 量级或更低. 常规的中子活化分析, 如采用 Se-77 $\mathrm{m}\left(T_{1 / 2}=17.6 \mathrm{~s}\right)$ 的短寿命 活化分析, 其灵敏度为 $5 \mathrm{ng}$ 左右, 一般长照活化 (用 Se-75, $T_{1 / 2}=120 \mathrm{~d}$ ) 灵敏度约为 0.1 $n g^{[3,4]}$, 难以满足上述极低含量 $\mathrm{Se}$ 的测定. 本工作采取加长照射时间和测量时间及降低本底 的办法来提高方法灵敏度.

本文报告了利用中子活化分析与聚丙烯酰胺凝胶电泳(PAGE) 技术相结合, 对水培实验 小麦幼苗过氧化物酶同工酶谱带中硒的含量和分布进行了分析. 目的是研究植物体内 POD 同工酶是否是硒酶? 外源性硒能否掺入 POD 同工酶及其对植物的生理功能影响等.

\section{1 实验}

(1)植物材料及培养方法. 冬小麦 (Triticum aestivum L. ) “H-65”品种, 由中国农业科学

2 Tanaka K. Saito H. Analysis of motion of the visual field by direction, expansion/contraction, and rotation cells clustered in the dorsal part of the medial superior temporal area of the macaque monkey. J Neurophysiol, 1989, 62:626 641

3 Duffy C J, Wurtz R H. Sensitivity of MST neurons to optic flow stimuli I . A continuum of response selectivity to large-field stimuli. J Neurophysiol, 1991, 65: 1 329 1 345

4 Sherk H, Kim J, Mulligan K. Are the preferred directions of neurons in cat extrastriate cortex related to optic flow? Visual Neuroscience, 1995, 12:887-894

5 Lagac I., Haes S, Raiguel S, et al. Responses of acaque STS neurons to optic flow components: a comparison of areas MT and MST. J Neurophysiol, 1994, 71:1597 1626 